\title{
Ruolo della chirurgia bariatrica nel paziente con malattia renale cronica
}

\author{
Rosa Grimaldi ${ }^{1}$, Maria Luisa Muci ${ }^{1}$, Silverio Rotondi ${ }^{1}$, Lida Tartaglione ${ }^{1}$, Mario Rizzello ${ }^{2}$, Francesca Abbatini ${ }^{2}$, \\ Gianfranco Silecchia², Sandro Mazzaferro ${ }^{1}$ \\ ${ }^{1}$ Dipartimento di Scienze Cardiovascolari Respiratorie Nefrologiche e Geriatriche, Polo Pontino Sapienza Università di Roma, Roma \\ ${ }^{2}$ Dipartimento di Scienze e Biotecnologie Medico Chirurgiche, Polo Pontino Sapienza Università di Roma, Roma
}

\begin{abstract}
Role of bariatric surgery in chronic kidney disease patients
Bariatric surgery represents the elective treatment of severe obesity $\left(B M I \geq 40 \mathrm{Kg} / \mathrm{m}^{2}\right)$ since it results in better control of cardiovascular risk factors and comorbidities typically associated with obesity, like diabetes, hypertension and dyslipidemia. Obesity is a recognized independent risk factor for chronic kidney disease and for progression of renal insufficiency. Pathomechanisms are still incompletely known. Obesity is associated with hyper-filtration, microalbuminuria and proteinuria all of which favor renal disease and its progression. This narrative review reports on the available evidence linking bariatric surgery and renal function since this surgery may affect proteinuria, hyper-filtration and glomerular filtration rate. Although available data are limited in particular in cases with more advanced stages of renal failure, bariatric surgery associates with improved filtration and lower proteinuria in patients with mild to moderate renal insufficiency. In patients with more advanced stages of renal failure, surgery should be considered if obesity represents a relative contraindication to transplantation. Surgery seems to improve graft survival. Further, nephrologists should be informed on the metabolic and nutritional changes associated with bariatric surgery, which could be responsible for untoward effects requiring early identification and treatment. Bariatric surgery could be a valid therapeutic option in renal patients to improve the negative clinical outcomes of obese subjects.
\end{abstract}

Keywords: Bariatric surgery, Chronic renal failure, Metabolic derangements, Progression of renal failure, Severe obesity

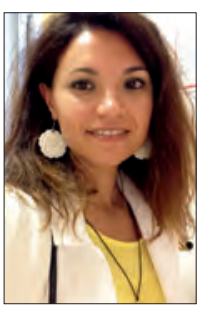

Rosa Grimaldi

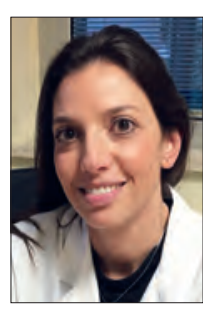

Maria Luisa Muci

\section{Introduzione}

L'obesità rappresenta un importante problema di salute pubblica e interessa più di un terzo della popolazione adulta mondiale. Non molto dissimili sono i
Accepted: October 28, 2016

Published online: December 2, 2016

Indirizzo per la corrispondenza:

Prof. Sandro Mazzaferro

Dipartimento di Scienze Cardiovascolari Respiratorie

Nefrologiche

Anestesiologiche e Geriatriche

Università degli Studi di Roma "La Sapienza"

V.le del Policlinico, 155

00185 Roma

sandro.mazzaferro@uniroma1.it dati epidemiologici italiani. L'Indagine Multiscopo Istat del 2012 dimostra che il 35.6\% della popolazione adulta italiana è in sovrappeso, mentre il $10.4 \%$ è obeso (BMI $>30 \mathrm{Kg} / \mathrm{m}^{2}$ ). Grande attenzione è rivolta anche alla popolazione pediatrica. I dati del 2014 forniti dal Sistema di Monitoraggio "Okkio alla Salute" indicano che i bambini in sovrappeso sono pari al $20.9 \%$, mentre quelli obesi sono pari al $9.8 \%$, compresi i bambini severamente obesi che, da soli, sono pari al $2.2 \%$ (1).

La prevalenza maggiore sembrerebbe essere registrata tra i gruppi con livelli socio-economici e culturali più bassi.

L'obesità è ormai riconosciuta come un fattore di rischio indipendente di malattia renale cronica e di progressione verso l'End Stage Renal Disease (ESRD). L'obesità rappresenta, quindi, un problema di salute pubblica su cui si è focalizzata l'attenzione dei clinici e di tutta la sanità, invitando e sponsorizzando una serie di campagne di sensibilizzazione volte a promuovere cambiamenti di stile di vita e alimentari per ridurre il più possibile il tasso di obesità della popolazione generale.

Il più delle volte, però, la terapia convenzionale non sembra dare risultati soddisfacenti. Paragonando le varie modalità di intervento volte alla riduzione del peso corporeo come la 
dieta ipocalorica, l'esercizio fisico, i cambiamenti dello stile di vita e la terapia farmacologica, queste hanno dimostrato di ottenere una percentuale di riduzione del peso corporeo molto bassa, non garantendo la stabilizzazione del peso a distanza di tempo. La chirurgia bariatrica, attualmente, rappresenta il trattamento d'elezione in caso di obesità severa, ottenendo risultati importanti in termini di efficacia e durata. Le attuali Linee Guida sull'obesità indicano che il trattamento bariatrico dovrebbe essere considerato in tutti i soggetti con obesità severa ( $\mathrm{BMI} \geq 40 \mathrm{Kg} / \mathrm{m}^{2}$ ) e in quelli con $\mathrm{BMI}>35 \mathrm{Kg} / \mathrm{m}^{2}$, in cui l'obesità si associa a importanti comorbidità $(2,3)$.

II trattamento chirurgico garantirebbe un'importante perdita di peso e il miglioramento delle condizioni cliniche e della qualità di vita, con riduzione della mortalità a lungo termine (4).

\section{Tecniche chirurgiche bariatriche}

Dal 1998, con la diffusione della laparoscopia, il numero di interventi chirurgici bariatrici eseguiti in tutto il mondo è aumentato del $761 \%$. In Italia, nel 2013, sono state eseguite circa 8.500 procedure di chirurgia bariatrica (5). Tali procedure si distinguono in: restrittive, metaboliche e malassorbitive (Tab. I).

Le procedure restrittive per eccellenza sono rappresentate dal Bendaggio Gastrico (Laparoscopic gastric banding, LB) regolabile (per anni l'intervento più eseguito al mondo e in Italia), la Gastroplastica Verticale, la Sleeve Gastrectomy (l'intervento più eseguito oggi in Italia) e, recentemente, la Gastric plication. L'effetto di questi interventi è quello di ridurre l'introito calorico per un effetto restrittivo gastrico determinato dall'apposizione di un "anello" intorno allo stomaco prossimale (Bendaggio Gastrico e Gastroplastica Verticale) o dalla gastroresezione reale (Sleeve Gastrectomy) o funzionale (Gastric Plication). II bypass gastrico (Laparoscopic Rouxen-Y gastric bypass, RYGB) consiste nell'esclusione dal tran- sito alimentare di gran parte dello stomaco, del duodeno e della prima parte di digiuno e rappresenta la principale procedura "metabolica". La tecnica malassorbitiva più eseguita e riconosciuta come "standard" è la diversione biliopancreatica (variante Scopinaro o Duodenal Switch), che associa una gastroresezione a un bypass intestinale vero e proprio. Il termine metabolico nasce dall'osservazione che già nell'immediato periodo post-operatorio si osservano miglioramenti dell'omeostasi glucidica e casi di "guarigione" dal diabete mellito di tipo II, indipendentemente dal calo ponderale (a cui, generalmente, segue un miglioramento delle comorbidità associate all'obesità patologica). Per cercare di spiegare questo effetto "metabolico", in questi ultimi dieci anni sono state proposte diverse teorie, anche se, a oggi, il vero meccanismo responsabile non è stato ancora identificato (6). Tutte le teorie proposte partono dall'assunto che la procedura di bypass dello stomaco, del duodeno e della prima porzione del digiuno determina una modificazione dell'assetto di alcuni entero-ormoni (Ghrelina, Peptide YY e il peptide glucagone simile GLP-1), che partecipano al mantenimento dell'omeostasi glucidica. Recentemente, un'altra procedura bariatrica restrittiva, la Sleeve Gastrectomy, si è dimostrata in grado di agire rapidamente, e indipendentemente dal calo ponderale, sull'omeostasi glucidica, determinando un miglioramento/guarigione dal diabete mellito di tipo II $(7,8)$. Come per il bypass gastrico, anche per la sleeve gastrectomy, procedura bariatrica sempre più diffusa, è stato invocato un meccanismo entero-ormonale alla base di queste modificazioni (9).

\section{Obesità e malattia renale cronica}

Studi di popolazione hanno ampiamente dimostrato lo stretto link tra obesità e malattia renale cronica.

I dati forniti dallo studio NHANES (National Health and

TABELLA I - Tecniche chirurgiche bariatriche, indicazioni e complicanze

\begin{tabular}{|c|c|c|c|}
\hline Procedura & $\begin{array}{l}\text { Tipologia di } \\
\text { intervento }\end{array}$ & Indicazioni & Complicanze \\
\hline Bendaggio Gastrico & Restrittivo & $\begin{array}{l}\text { BMI 35-45, compliance nutrizionale, no disturbi del } \\
\text { comportamento alimentare }\end{array}$ & Scivolamento, erosione \\
\hline Gastroplastica verticale & Restrittivo & $\begin{array}{l}\text { BMI 35-45, compliance nutrizionale, no disturbi del } \\
\text { comportamento alimentare }\end{array}$ & Fistola gastro-gastrica \\
\hline Sleeve Gastrectomy & Restrittivo & $\begin{array}{l}\text { BMI >35, compliance nutrizionale, no disturbi del } \\
\text { comportamento alimentare }\end{array}$ & Fistola gastrica \\
\hline Gastric Plication & Restrittivo & $\begin{array}{l}\text { BMI 35-45, compliance nutrizionale, no disturbi del } \\
\text { comportamento alimentare }\end{array}$ & Erniazione, occlusione gastrica \\
\hline $\begin{array}{l}\text { Gastric bypass } \\
\text { (con le sue varianti) }\end{array}$ & Metabolico & $\begin{array}{l}\text { BMI } 35-45 \text {, no disturbi del comportamento alimen- } \\
\text { tare, diabete di lunga data (<10 anni), esofagite }> \\
\text { classe A }\end{array}$ & $\begin{array}{l}\text { Fistola anastomotica, ernia interna, } \\
\text { carenza (rara) di ferro e di vitamine } \\
\text { del gruppo B }\end{array}$ \\
\hline $\begin{array}{l}\text { Diversione } \\
\text { bilio-pancreatica }\end{array}$ & Malassorbitivo & BMI >35, scarsa compliance nutrizionale & $\begin{array}{l}\text { Fistola anastomotica, ernia interna, } \\
\text { carenza (frequente) di ferro, calcio } \\
\text { e vitamine del gruppo B }\end{array}$ \\
\hline
\end{tabular}


Nutrition Examination Survey) hanno evidenziato un incremento parallelo della prevalenza di obesità e malattia renale cronica avanzata, suggerendo che l'obesità rappresenta uno dei fattori di rischio più importanti di danno renale (10).

In particolare, è stata osservata una correlazione tra obesità e sviluppo di glomerulosclerosi focale e segmentale (GSFS). Analisi retrospettive condotte su biopsie renali di soggetti obesi hanno evidenziato che il quadro di GSFS associato all'obesità si differenzia dalla forma idiopatica per la bassa frequenza di sindrome nefrosica, la presenza di poche lesioni segmentali e le aumentate dimensioni dei glomeruli (11).

Il panorama di lesioni istologiche riscontrabili in caso di obesità è, però, molto variabile. Esse comprendono aumento della matrice mesangiale, proliferazione delle cellule mesangiali, ipertrofia dei podociti e glomerulomegalia, sia isolati che associati a vari gradi di glomerulosclerosi focale e segmentale (12). Tali lesioni istologiche sembrano instaurarsi precocemente, prima del riscontro clinico di una riduzione del filtrato glomerulare, come dimostrato dall'analisi di biopsie renali eseguite in soggetti obesi con funzione renale normale (clearance della creatinina $>90 \mathrm{~mL} / \mathrm{min}$ ) (13).

La principale alterazione renale riscontrata in associazione con l'obesità è la proteinuria. È stata dimostrata una correlazione significativa tra elevato Body Mass Index (BMI) e proteinuria, marker di danno glomerulare, in soggetti in sovrappeso sia diabetici che non diabetici.

Dati clinici e sperimentali dimostrano che l'obesità induce un'iperfiltrazione glomerulare attraverso diversi meccanismi fisiopatologici, alcuni dei quali non perfettamente noti. L'iperfiltrazione glomerulare determina microalbuminuria e proteinuria, favorendo la comparsa o la progressione di un danno renale preesistente (14).

È noto da tempo che l'obesità si associa a un aumentato rischio di diabete mellito di tipo II, ipertensione arteriosa, malattie cardiovascolari, dislipidemia e sindrome metabolica, tutti potenzialmente coinvolti nell'eziopatogenesi e nella progressione della malattia renale cronica (15). In particolare, nei soggetti diabetici, l'obesità incrementa il rischio di DKD (Diabetic Kidney Disease) mediante meccanismi fisiopatologici complessi. Pertanto, il trattamento dell'obesità appare di fondamentale importanza, permettendo di ridurre le comorbidità ad essa associate, con un potenziale effetto positivo sulla prevenzione del danno renale $(16,17)$.

\section{Meccanismi fisiopatologici di danno renale correlati all'obesità}

I meccanismi fisiopatologici responsabili del danno renale in corso di obesità sono diversi e complessi e alcuni ancora non perfettamente chiari. Tra i principali vengono menzionati la resistenza insulinica, l'iperinsulinemia, l'infiammazione e l'attivazione del sistema renina-angiotensina-aldosterone (Fig. 1).

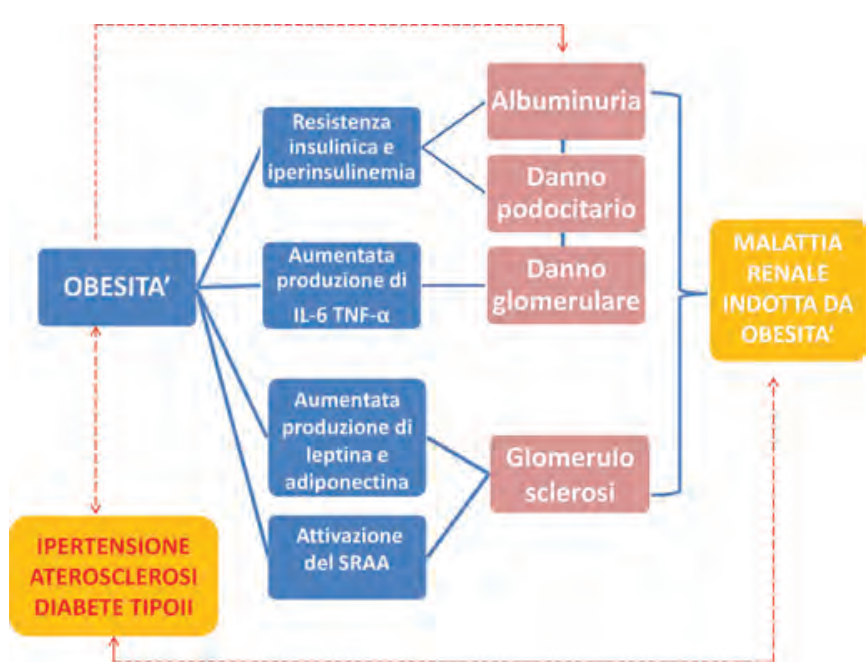

Fig. 1 - Meccanismi fisiopatologici di danno renale correlati all'obesità.

\section{Resistenza insulinica e iperinsulinemia}

Alcune evidenze sperimentali suggeriscono che l'insulina possa avere un ruolo nella patogenesi del danno renale. In condizioni normali, l'insulina, interagendo con il suo recettore, induce la fosforilazione del substrato insulinico-1 (IRS1), stimolando l'attivazione della fosfatidilinositol-3-kinasi (PI3-Kinasi). Tale kinasi inibisce la protein-kinasi B, la quale, mediante la ridistribuzione della proteine di trasporto del glucosio GLUT1 e GLUT4, modula il trasporto cellulare del glucosio. L'attivazione della PI3-Kinasi risulta cruciale per tutti i meccanismi di trasduzione del segnale sia nel rene che in tutti i tessuti sensibili all'insulina. Alterazioni a carico dei meccanismi del segnale pre-recettoriali, recettoriali e post-recettoriali sono responsabili della comparsa di resistenza all'azione dell'insulina a carico degli organi bersaglio e della presenza di iperinsulinemia compensatoria. Già nel 1980, Mogensen et al ipotizzarono che la barriera glomerulare risultasse sensibile all'insulina. Indagini più recenti hanno evidenziato che la resistenza insulinica e l'iperinsulinemia conducono alla comparsa di albuminuria prima ancora dell'instaurarsi delle alterazioni strutturali a livello renale. Pertanto, tali condizioni patologiche sembrano giocare un ruolo centrale nella genesi del danno renale $(18,19)$.

Di recente, si è focalizzata l'attenzione sulla principale struttura della barriera glomerulare, i podociti. Studi in vitro condotti su linee cellulari di podociti umani immortalizzati hanno evidenziato che, in seguito allo stimolo insulinico, $i$ podociti rispondono raddoppiando il trasporto di glucosio attraverso la trasposizione dei trasportatori del glucosio GLUT1 e GLUT4 dai vacuoli intracellulari alla superficie di membrana podocitaria. Tale processo vede coinvolti i filamenti di actina del citoscheletro, i quali, in seguito all'attivazione delle vie di trasduzione del segnale intracellulare, modificherebbero il loro assetto strutturale per facilitare l'esposizione delle pro- 
teine di trasporto del glucosio. Le alterazioni di trasduzione del segnale intracellulare, oltre a modificare il trasporto del glucosio, potrebbero indurre delle alterazioni strutturali del citoscheletro dei podociti tali da causare la perdita della funzione di barriera glomerulare e la comparsa di proteinuria. Pertanto, alterazioni strutturali del citoscheletro dei podociti potrebbero avere un ruolo chiave nelle perdita della barriera di filtrazione glomerulare e nella comparsa di nefropatia diabetica o di albuminuria secondaria a resistenza insulinica e a iperinsulinemia (20).

Inoltre, è noto che l'insulina stimola la proliferazione delle cellule mesangiali e la sintesi di proteine che regolano la deposizione della matrice mesangiale e della membrana basale glomerulare (21).

L'insulina potrebbe contribuire alla comparsa di microalbuminuria e "Chronic Kidney Disease" (CKD) mediante attivazione del TGF- $\beta$, il quale induce fibrosi interstiziale e un rimodellamento podocitario con conseguente danno della barriera di filtrazione glomerulare e comparsa di microalbuminuria (22).

\section{Infiammazione}

Il tessuto adiposo rappresenta un sito di produzione di citochine infiammatorie e fattori di crescita coinvolti nello sviluppo di danno renale nei soggetti obesi (23).

In particolare, in entrambe le condizioni di obesità e diabete, i macrofagi infiltranti il tessuto adiposo viscerale producono elevati livelli di TNF- $\alpha$, il quale stimola la produzione di IL-6.

L'IL-6 interviene nella regolazione delle proteine di fase acuta, tra cui l'attivatore del plasminogeno-1 (PAI) e il fibrinogeno, entrambi implicati nei processi pro-infiammatori e pro-trombotici in corso di proteinuria. II riscontro di elevati livelli di TNF- $\alpha$ e IL-6 espressi a livello glomerulare in pazienti obesi farebbe, quindi, ipotizzare un loro ruolo nei meccanismi di danno renale in corso di obesità (24).

Inoltre, la deposizione di acidi grassi liberi a livello tissutale conduce a un accumulo di metaboliti tossici. La lipotossicità indotta dagli acidi grassi liberi legati all'albumina sembrerebbe responsabile della comparsa di infiammazione tubulo-interstiziale e di fibrosi (25).

\section{Sistema Renina-Angiotensina-Aldosterone}

Fattori come l'ipertensione intraglomerulare e lo stretch meccanico inducono in vitro la produzione di Angiotensina II (ATII) a livello tissutale. Anche l'iperglicemia e l'iperinsulinemia sembrano stimolare la produzione di Angiotensina II nei podociti.

Nei soggetti obesi, gli elevati livelli di ATII e l'aumentata espressione del suo recettore AT1 causano un netto incremento della vasocostrizione arteriolare e aumento della pressione e della filtrazione glomerulare, potenziali respon- sabili di glomerulosclerosi. Inoltre, il grasso viscerale produce angiotensinogeno potenziando l'attivazione del SRAA (26).

L'aldosterone modula l'attivazione della NADPH ossidasi e la generazione di specie reattive dell'ossigeno (ROS), come dimostrato da uno studio condotto su ratti, nei quali l'infusione diretta di aldosterone induce danno podocitario e albuminuria. Tale effetto si riduce con la somministrazione di anti-aldosteronici e di inibitori $\operatorname{ACE} \operatorname{ARB}(27,28)$.

\section{Fattori ormonali: LEPTINA e ADIPONECTINA}

Nei soggetti obesi, gli elevati livelli di leptina stimolano la produzione di TGF- $\beta 1$ da parte dell'endotelio. Recenti evidenze indicano che questo fattore di crescita pro-fibrotico agirebbe per via paracrina a livello mesangiale, stimolando la produzione di collagene di tipo IV e la proliferazione delle cellule endoteliali glomerulari, intervenendo, in questo modo, nei meccanismi di glomerulosclerosi $(29,30)$.

Contestualmente, i ridotti livelli di adiponectina riscontrati nei soggetti obesi sono correlati negativamente con l'escrezione urinaria di albumina. II trattamento con adiponectina nell'animale da esperimento si associa a una protezione della funzione podocitaria e alla riduzione della proteinuria (31).

\section{Altri fattori}

Altri fattori di tipo emodinamico potrebbero essere coinvolti nel meccanismo di danno renale in corso di obesità. Per esempio, l'aumentata pressione intra-addominale dovuta al grasso viscerale può essere responsabile di stasi venosa a livello renale, con conseguente alterazione delle pressioni glomerulari e comparsa di albuminuria $(32,33)$.

\section{Effetti della chirurgia bariatrica nei diabetici}

Il termine "diabesity" è stato introdotto di recente nella letteratura corrente per descrivere la frequente coesistenza di diabete mellito e obesità. È stato in precedenza sottolineato come, nei soggetti diabetici, l'obesità incrementi il rischio di DKD mediante meccanismi fisiopatologici complessi e quanto sia di fondamentale importanza il trattamento chirurgico.

Diversi studi hanno documentato una remissione del diabete mellito di tipo II nel 37-95\% dei pazienti, associato a un miglioramento dei parametri metabolici e alla riduzione della mortalità a lungo termine, in seguito al trattamento chirurgico bariatrico. Ponendo a confronto le varie tecniche chirurgiche rispetto al profilo di sicurezza, al raggiungimento del calo ponderale e alle complicanze associate, pazienti trattati con RYGB a distanza di 1 anno dall'intervento mostrano un maggiore calo ponderale, la risoluzione del diabete e un miglioramento della qualità di vita rispetto a quelli trattati con LB. Sebbene la RYGB sia considerata la procedura chirurgica più complessa, in pazienti selezionati e preparati rappresenta il trattamento di elezione in termini di miglior profilo di 
rischio-beneficio e di una più alta percentuale di risoluzione del diabete (34).

La presenza di diabete scompensato in soggetti con obesità moderata potrebbe rappresentare un'indicazione al trattamento chirurgico, come dimostrato da Lee in un trial clinico randomizzato e controllato in cui si è raggiunta una remissione nel $93 \%$ dei soggetti diabetici con obesità moderata trattati con RYGB e nel $47 \%$ dei soggetti sottoposti a sleeve gastrectomy, osservando un miglioramento della sindrome metabolica a distanza di 1 anno dal trattamento (35). Studi osservazionali a lungo termine hanno confermato remissione del DM dopo 2 anni dal trattamento chirurgico nel $72.3 \%$ dei pazienti trattati rispetto al $16 \%$ del gruppo controllo. La chirurgia bariatrica si associa a remissione del diabete, a una ridotta incidenza di IMA non solo nei pazienti obesi in generale ma anche nei soggetti diabetici e a effetti positivi sull'albuminuria. Vi è, inoltre, l'evidenza, nel lungo termine, di una ridotta incidenza di complicanze micro e macro-vascolari dopo trattamento chirurgico, indipendentemente dalla procedura impiegata (36).

\section{Effetti della perdita di peso sulla funzione renale nei pazienti obesi con CKD}

Gli effetti della chirurgia bariatrica sulla funzionalità renale sono stati a lungo oggetto di discussione. La maggior parte degli studi pubblicati in passato era condotta su pazienti con normale funzione renale, mentre altri presentavano dati incompleti e contrastanti.

Nel 1980, Brochner et al dimostrarono per la prima volta che i pazienti obesi sottoposti a bypass intestinale presentavano una riduzione del GFR a distanza di 1 anno dall'intervento (37).

Risultati simili furono ottenuti da altri due studi condotti da Changas e Serra, i quali osservavano analogamente una riduzione del GFR in pazienti sottoposti a chirurgia bariatrica dopo 1 anno dall'intervento $(38,39)$.

Uno studio prospettico pubblicato da Diaz nel 2006 ha valutato gli effetti della riduzione del peso corporeo sia sulla pressione arteriosa che sui parametri di funzione renale in 61 soggetti obesi sottoposti a chirurgia bariatrica. Tutti i pazienti arruolati presentavano valori di creatinina nei limiti. II gruppo di pazienti sottoposti a chirurgia bariatrica presentava, a 12 mesi dall'intervento, una riduzione significativa della PAS e PAD. Inoltre, si registrava una riduzione della clearance della creatinina, della proteinuria e dell'albuminuria delle 24 ore nonché della microematuria. A distanza di 24 mesi, i pazienti continuavano a perdere peso, anche se in proporzione inferiore rispetto al $1^{\circ}$ anno di follow-up. Nel secondo anno di follow-up si osservava un'ulteriore riduzione dell'albuminuria delle 24 ore, mentre gli altri parametri rimanevano simili al primo anno di osservazione. Tale studio, pur documentando in maniera analoga una riduzione del filtrato glomerulare in pazienti sottoposti a chirurgia bariatrica, in realtà dimostra che la riduzione del GFR osservata è da attribuire alla ridotta iperfiltrazione glomerulare, che si traduce, quindi, in una riduzione della proteinuria che potrebbe rallentare l'evoluzione del danno renale associato all'obesità (40).

Una recente review condotta da Bolignano D. et al. ha confrontato gli effetti delle differenti modalità di intervento nei soggetti obesi sull'outcome renale (41).

Rispetto all'andamento della funzione renale, nella maggioranza degli studi analizzati si osservano una stabilità dei valori di eGFR o un lieve miglioramento in pazienti con preesistente insufficienza renale cronica moderata (42); in altri casi, si può ottenere una normalizzazione dei valori di eGFR come risultato della ridotta iperfiltrazione glomerulare (43).

In particolare, viene riportato uno studio condotto da Alexander nel 2009 nel quale sono stati arruolati anche pazienti con malattia renale cronica in fase avanzata, di cui 2 erano in trattamento emodialitico sostitutivo. Oltre a documentare un miglioramento di tutti i parametri clinici, si è registrato un miglioramento della funzionalità renale. In particolare, i due pazienti in trattamento emodialitico hanno sospeso la terapia sostitutiva rispettivamente per 7 e 27 mesi. Inoltre, in un caso è stata documentata la risoluzione della glomerulonefrite membranosa (MGN). I restanti pazienti hanno presentato una stabilità della funzione renale nel periodo di follow-up di 2-5 anni (44). Anche lo studio condotto da Navaneethan che includeva una piccola sottopopolazione di circa 25 soggetti obesi con IRC al III stadio documentava un incremento dell'eGFR da 47.9 a $61.6 \mathrm{~mL} /$ $\mathrm{min} / 1.73 \mathrm{~m}^{2}$ a distanza di 1 anno (42).

Risultati contrastanti sono stati riportati da Schuster nel 2011, il quale ha arruolato ben 813 soggetti di cui 56 con insufficienza renale lieve e moderata. Dopo chirurgia bariatrica i valori di creatinina risultavano ridotti nei pazienti con IRC di grado lieve ( $\mathrm{s} C r$ 1.3-1.6 mg/dL) e aumentati in quelli con IRC di grado moderato $(\mathrm{s} C \mathrm{Cr}>1.6 \mathrm{mg} / \mathrm{dL})(45)$.

Uno studio svedese, S.O.S. study (Swedish Obesity Subject study), ha valutato gli effetti a lungo tempo della chirurgia bariatrica rispetto alla terapia classica (gruppo controllo) sull'incidenza di albuminuria, evidenziandone una riduzione del $50 \%$ rispetto al gruppo controllo (HR $0.46,95 \% \mathrm{Cl} 0.37-0.57$, $\mathrm{p}<0.001$ ) e dimostrando, per la prima volta, che il trattamento bariatrico si associa a una riduzione dell'incidenza annua di albuminuria nei soggetti obesi (46).

Ancora più controverso è l'approccio ai pazienti obesi in trattamento dialitico. Dati Europei basati su 1271 pazienti incidenti in dialisi mostrano che l'obesità è fortemente associata a un elevato rischio di perdita della funzione renale residua dopo I'inizio della terapia dialitica. Inoltre, studi osservazionali hanno evidenziato un outcome migliore dei soggetti obesi nella popolazione dialitica. Ciò ha indotto i clinici a promuovere un incremento di peso corporeo nei soggetti in trattamento dialitico. Infatti, l'obesità si potrebbe associare a una migliore mobilizzazione di cellule staminali, a una migliore eliminazione delle tossine uremiche lipofile, a un miglio- 
re tenore osseo e a una migliore tolleranza emodinamica. In aggiunta, l'aumentata riserva adiposa in genere riflette una migliore riserva energetica e una conservata appetenza, prevenendo per un lungo periodo gli effetti catabolici associati alla malattia renale terminale. In realtà tale generalizzazione non è appropriata, in quanto sono necessarie ulteriori considerazioni riguardo ad alcune caratteristiche fenotipiche di tali pazienti. Infatti, a differenza dei soggetti obesi anziani, quelli più giovani hanno un rischio di morte maggiore in presenza rispettivamente di un più basso o di un più elevato BMI. Quindi, mentre, negli anziani in dialisi, l'obesità sembra essere protettiva rispetto alla mortalità, nei soggetti giovani l'obesità rappresenta un fattore di rischio.

Uno studio pubblicato nel 2012 da Luaces ha valutato gli effetti della chirurgia bariatrica sia sulla funzione renale che su quella cardiaca. A distanza di 1 anno dall'intervento, i pazienti arruolati hanno presentato cambiamenti favorevoli nella geometria sia del ventricolo sinistro che dello stato emodinamico, con riduzione dello spessore relativo di parete (RWT) e dell'indice di massa ventricolare sinistra (LVMI). Inoltre, a distanza di 1 anno, non si sono osservate riduzioni significative dell'eGFR nei vari gruppi di trattamento, sebbene si sia registrato un miglioramento dello stato di iperfiltrazione glomerulare passando dal $14.8 \%$ al $9.8 \%$ dei pazienti. La percentuale di pazienti con funzione renale compromessa (eGFR $<60 \mathrm{~mL} / \mathrm{min}$ ) si è ridotta dall' $8.3 \%$ al 3.3\% dopo 1 anno di follow-up, dimostrando un netto miglioramento del filtrato glomerulare (47).

\section{Complicanze metaboliche}

\section{Iperossaluria e calcolosi renale}

Di recente, si è focalizzata l'attenzione sugli effetti negativi a lungo termine della chirurgia bariatrica. In particolare, si è cercato di chiarire quali siano i cambiamenti metabolici ai quali i soggetti trattati vanno incontro. La riduzione dell'introito alimentare, spesso osservata in seguito al trattamento chirurgico bariatrico, può condurre a malnutrizione severa, anemia, perdita della massa ossea, malnutrizione proteica, neuropatia periferica ed encefalopatia. Tali complicanze possono essere più o meno presenti, manifestandosi con quadri di severità differenti in base alle diverse tecniche chirurgiche adoperate.

Nello spettro del malassorbimento di grassi e di acidi biliari nei pazienti sottoposti a chirurgia, è frequente lo sviluppo di iperossaluria enterica e di calcolosi da ossalato di calcio. Il malassorbimento di grassi che conduce a steatorrea gioca un ruolo centrale nello sviluppo dell'iperossaluria enterica. L'eccesso di acidi grassi liberi nel lume intestinale induce saponificazione del calcio intestinale impedendone il legame con l'ossalato. L'ossalato così libero viene assorbito in grandi quantità dalla mucosa intestinale a livello del colon. Inoltre, la permeabilità della mucosa risulta ulteriormente aumentata dallo stato infiammatorio indotto dagli acidi grassi liberi e dai sali biliari. L'aumentato assorbimento intestinale di ossalato è responsabile della comparsa di iperossaluria. Il riscontro di iperossaluria a seguito di un bypass digiuno-ileale, pertanto, è molto frequente. Tale intervento è stato ormai abbandonato, in quanto gravato da molte complicanze. Rispetto ai nuovi tipi di metodi chirurgici, il bypass gastrico alla Roux-en-Y è, attualmente, quello più comunemente utilizzato. Recenti dati dimostrano che tale procedura rappresenta una condizione potenziale per lo sviluppo di iperossaluria enterica e nefrolitiasi, anche se in maniera inferiore rispetto alla diversione biliopancreatica, mentre le procedure restrittive come il bendaggio gastrico e la Sleeve gastrectomy presentano un rischio litogeno nettamente inferiore rispetto alle precedenti (48).

L'incidenza di calcolosi renale aumenta già a partire dai primi 2 anni successivi alla procedura chirurgica, arrivando, dopo 10 anni di follow-up, al 14\% nei soggetti sottoposti a RYGB rispetto al 7\% dei soggetti obesi non trattati. Nel $94 \%$ dei casi, i calcoli sono costituiti da CaOx (49).

L'iperossaluria enterica che si osserva dopo RYGB non è responsabile solo della comparsa di calcolosi renale. Ormai sono diverse le descrizioni di un nuovo onset di malattia renale descritta come nefropatia interstiziale ossalica. Diversi pazienti con iperossaluria enterica dopo chirurgia bariatrica hanno mostrato un progressivo peggioramento della funzione renale con possibile evoluzione verso la malattia renale avanzata. Male et al. hanno descritto ben 26 casi di insufficienza renale a seguito di bypass digiuno-ileale. In 17 casi su 26, era disponibile l'esame istologico che documentava la presenza di cristalli di ossalato di calcio a livello tubulare accompagnata da nefrite interstiziale cronica. Anche Asplin e Coe hanno evidenziato che i soggetti che presentavano calcoli di ossalato di calcio avevano elevati livelli di ossalato nelle urine (in media $100 \mathrm{mg} / \mathrm{die}$ ). Inoltre, l'aumentata concentrazione intratubulare di ossalato si associava a un aumentato rischio di nefropatia ossalica, evidenziando una progressione verso la malattia renale avanzata nell'arco di 3 mesi nel $72.7 \%$ dei soggetti sottoposti a chirurgia bariatrica (50). Successivamente, Nasr ha identificato ben 11 casi di nefropatia ossalica dopo RYGB, mostrando quadri di danno tubulo-interstiziale sia acuto che cronico con abbondante deposizione di cristalli di ossalato di calcio a livello tubulare (51).

Oltre all'iperossaluria, è possibile riscontrare altre alterazioni urinarie come riduzione del volume urinario e ipocitraturia, entrambe responsabili di una maggiore sovrasaturazione di ossalato di calcio nelle urine. La ridotta citraturia riflette, probabilmente, la presenza di acidosi metabolica sistemica frequentemente riscontrata dopo chirurgia bariatrica. Inoltre l'acidosi metabolica cronica secondaria alla perdita cronica di bicarbonato a livello gastrointestinale può essere responsabile degli effetti sull'asse osso-rene, determinando demineralizzazione ossea e osteopenia-osteoporosi.

Anche le alterazioni della flora batterica intestinale potrebbero giocare un ruolo importante nella genesi dell'ipe- 
rossaluria enterica. In particolare, l'Oxalobacter formigenes, comune commensale anaerobico capace di metabolizzare l'acido ossalico, è stato associato a una riduzione del $70 \%$ del rischio di calcolosi ricorrente da ossalato di calcio. Pertanto, le modifiche della normale flora batterica intestinale potrebbero essere responsabili di una maggiore disponibilità intestinale di ossalato, conducendo a iperossaluria. Ciò suggerisce che, nei pazienti sottoposti a RYGB, una più alta percentuale di colonizzazione intestinale da parte dell'O. formigenes potrebbe determinare una riduzione dei livelli di ossalato nell'intestino riducendone, di conseguenza, la concentrazione nelle urine (52).

Tali dati sottolineano la necessità di uno screening e di un monitoraggio attenti nei soggetti sottoposti a chirurgia bariatrica, in particolare in quelli sottoposti a RYGB, al fine di identificare rapidamente le alterazioni funzionali e metaboliche a cui vanno incontro e di apportare misure dietetiche e di intervento precoci per correggerle.

In particolare, il riscontro di un rapido peggioramento della funzione renale in pazienti con recente RYGB deve far ipotizzare la comparsa di un'incipiente nefropatia ossalica, rendendo necessario un adeguato approfondimento diagnostico.

\section{Anemia}

Si stima che la prevalenza di anemia in soggetti candidati alla chirurgia bariatrica sia pari al $10-15 \%$, più elevata rispetto alla popolazione generale, mentre la prevalenza della carenza di ferro (con o senza anemia) può essere superiore (30-40\%). A oggi, vi sono ancora pochi studi rispetto alla valutazione dello stato nutrizionale del soggetto candidato alla chirurgia bariatrica. Tuttavia, è noto che lo stato infiammatorio correlato all'obesità, mediante attivazione del sistema immunitario, determina alterazioni nell'omeostasi del ferro inducendo iposideremia, alterata eritropoiesi e anemia di lieve o moderata entità. Per tali motivi, non è infrequente riscontrare deficit di alcuni nutrienti prima del trattamento chirurgico. Generalmente, obesità e sovrappeso sono fattori di rischio per lo sviluppo di carenze nutrizionali, in particolare il deficit di ferro è stato riportato nel 35\% circa dei casi. L'anemia peri-operatoria è stata, inoltre, associata a una morbidità e a una mortalità aumentate, con peggioramento della qualità di vita; al contrario, il trattamento dell'anemia e delle carenze marziali sembra migliorare l'outcome e la qualità di vita. Sulla base di tali premesse, l'assetto marziale dovrebbe essere attentamente e preventivamente valutato (attraverso il monitoraggio dei parametri biochimici) in tutti i soggetti candidati a qualunque procedura di chirurgia bariatrica, con particolare attenzione nei confronti di donne in età fertile o in gravidanza e degli adolescenti (53).

\section{Deficit vitaminici}

Fino a qualche anno fa non vi erano Linee Guida sul followup nutrizionale dei pazienti sottoposti a chirurgia bariatrica.
Inoltre, nella maggioranza dei casi, le supplementazioni multivitaminiche standard si sono dimostrate non sufficienti per correggere i deficit nutrizionali a cui tali soggetti vanno incontro.

II deficit di vitamina B12 è estremamente frequente dopo bypass gastrico a causa dell'inadeguata secrezione di fattore intrinseco, mentre, in seguito a interventi di derivazione biliopancreatica con bypass duodenale, il malassorbimento dei grassi è responsabile del ridotto assorbimento di vitamine liposolubili come le vitamine A e D. Inoltre, il vomito e la crescita di batteri del piccolo intestino sembrano responsabili della comparsa di encefalopatia di Werniche secondaria al deficit di tiamina (vitamina B1), mentre lo stato infiammatorio cronico di tali pazienti, come documentato dal riscontro di elevati livelli di proteina $\mathrm{C}$ reattiva, si associa a bassi livelli di vitamina B6 e vitamina C (54).

Nonostante venga fornita, il più delle volte, un'adeguata supplementazione di vitamine standard, nei pazienti trattati chirurgicamente si riscontrano livelli vitaminici variabili. Ponendo a confronto i soggetti sottoposti rispettivamente a bypass duodenale e gastrico è stata osservata una riduzione dei livelli sierici di vitamina $A$ in entrambi i gruppi di trattamento a 6 settimane, a 6 mesi e a 1 anno dall'intervento. I livelli di vitamina $\mathrm{E}$ apparentemente ridotti, se aggiustati per la concentrazione di lipidi sierici, risultavano aumentati a distanza di 1 anno dall'intervento.

Per la vitamina D, i dati sono contrastanti, dato che, in circa l' $80 \%$ dei pazienti in fase sia pre- che post-operatoria, è stata riscontrata un'ipovitaminosi $D$ con iperparatiroidismo secondario (55).

\section{Metabolismo osseo}

Le modifiche anatomiche indotte dall'intervento bariatrico determinano il malassorbimento di diversi nutrienti, inclusa la vitamina $D$. Tale deficit vitaminico è comunemente presente nei soggetti sottoposti a RYGB. Ciò riduce l'assorbimento intestinale di calcio con conseguente iperparatiroidismo secondario e perdita di massa ossea. In realtà, la riduzione della massa ossea sembra osservarsi nonostante le supplementazioni di vitamina $D$, indicando che il deficit di vitamina $D$ potrebbe non esserne l'unico responsabile (56).

Studi sperimentali hanno dimostrato una riduzione del BMD in ratti sottoposti a RYGB già dopo 2 settimane dall'intervento, che persiste fino a 14 settimane di osservazione, con riduzione sia del volume osseo che della trabecolatura ossea vertebrale e tibiale. Inoltre, il riscontro di un quadro di acidosi metabolica con aumentati livelli sierici di lattato ed elevata calciuria suggerisce che l'iniziale malassorbimento di calcio può avere un ruolo chiave nella riduzione della massa ossea (57).

\section{Chirurgia bariatrica e trapianto renale}

La presenza di obesità ha rappresentato fino a poco tempo fa una controindicazione al trapianto renale. La chirurgia 
bariatrica è stata attualmente introdotta come "ponte" per i soggetti obesi diabetici candidati al trapianto renale.

Esistono studi prospettici multicentrici che hanno valutato l'impatto che ha l'obesità sul paziente ricevente il trapianto renale in considerazione delle complicanze che possono manifestarsi a breve e a lungo termine, come le malattie cardiovascolari e respiratorie e il diabete mellito (58).

L'obesità può influenzare l'outcome del trapianto attraverso diversi meccanismi, alcuni dei quali comprendono meccanismi patofisiologici simili a quelli che si riscontrano sui reni nativi dei soggetti obesi (59).

Confrontando i pazienti trapiantati obesi e quelli con normale BMI, in una recente meta-analisi (138.081 pazienti) la presenza di obesità si associa a un aumento del $68 \%$ del rischio di Delayed Graft Function (DGF). II DGF è stato attribuito alla concorrenza di danno ischemico e immunologico. L'obesità potrebbe causare DGF attraverso una combinazione di meccanismi come la vasocostrizione da aumentata attività del sistema nervoso simpatico e il protratto tempo operatorio.

A tal proposito, l'elevata incidenza di complicanze mediche e chirurgiche post-operatorie che è stata osservata nei soggetti obesi che ricevono il trapianto potrebbe aumentare la probabilità di sviluppare $\operatorname{DGF}(59,60)$.

Vi è, inoltre, una maggiore incidenza nei soggetti obesi di complicanze peri-operatorie anestesiologiche e infettive con prolungata ospedalizzazione rispetto ai soggetti non obesi sottoposti a trattamento chirurgico. Sebbene non siano state riscontrate differenze significative nel rischio di morte tra soggetti trapiantati obesi e con normale BMI, questo suggerisce che i soggetti obesi potrebbero non essere esclusi dalle liste d'attesa di trapianto unicamente sulla base dell'obesità; tuttavia, i soggetti obesi che ricevono un trapianto di rene hanno un rischio marginalmente più alto di morte associata a perdita del graft e allo sviluppo di DGF.

In aggiunta, ci sono evidenze che l'obesità potrebbe alterare il metabolismo e la biodisponibilità di farmaci immunosoppressori esponendo il rene trapiantato a un danno immunologico cronico.

Pertanto, i soggetti obesi che ricevono il trapianto renale dovrebbero essere attentamente e preventivamente valutati per minimizzare la mortalità peri-operatoria e per ridurre il rischio di danni aggiuntivi al rene trapiantato.

Rispetto al ruolo della chirurgia, esperienze preliminari basate su recenti case report su pazienti con BMI superiore a $35 \mathrm{~kg} / \mathrm{m}^{2}$ con malattia renale cronica sottoposti a trattamento bariatrico (RYGB) e, successivamente, a trapianto renale hanno confermato la completa sicurezza di questa procedura, associandosi a riduzione del peso corporeo e a un miglioramento in termini di parametri metabolici e di modifiche farmacologiche, con un effetto chiaramente positivo sulla riduzione delle complicanze legate alla procedura e sulla prolungata sopravvivenza del graft (60).

\section{Conclusioni}

L'obesità rappresenta un problema di salute pubblica mondiale ed è una causa di morbidità e mortalità.

È, ormai, ampiamente dimostrato il link esistente tra obesità e malattia renale cronica attraverso una serie di meccanismi fisiopatologici complessi. L'aumentata massa grassa non solo promuove la malattia renale cronica indirettamente attraverso l'ipertensione, l'aterosclerosi e il diabete, ma anche mediante meccanismi diretti, ancora solo in parte chiariti. Pertanto, le attuali tecniche chirurgiche bariatriche dovrebbero essere considerate quando la terapia convenzionale non produce risultati. Tali procedure si sono dimostrate efficaci non solo sulle alterazioni funzionali renali attraverso la riduzione della proteinuria e dell'iperfiltrazione, ma anche sul miglioramento del GFR a un anno dall'intervento, oltre ad aver permesso la riduzione delle complicanze legate alla procedura stessa e alla prolungata sopravvivenza del graft nei pazienti trapiantati.

Si assiste, inoltre, a una riduzione di tutte le comorbidità conseguente a un migliorato controllo glicemico nei pazienti obesi diabetici, al controllo dei valori pressori e al miglioramento della performance cardiaca con riduzione dell'ipertrofia ventricolare sinistra e della massa ventricolare, contribuendo a una ridotta mortalità a lungo termine in seguito al trattamento chirurgico (47).

Queste tecniche presentano, però, delle complicanze a lungo termine legate prevalentemente alle alterazioni metaboliche che si manifestano con quadri di diversa entità, come la calcolosi, i deficit vitaminici e l'anemia.

II trattamento dell'obesità richiede, dunque, un approccio di tipo multidisciplinare per garantire, oltre alla perdita di peso, un effetto positivo sulle comorbidità associate all'obesità e sul controllo della progressione del danno renale. Per ottenere conferma degli effetti del trattamento chirurgico bariatrico e chiarire i meccanismi fisiopatologici di danno renale in corso di obesità sono necessari studi con un follow-up più lungo e la valutazione di nuovi biomarker di danno glomerulare renale.

\section{Disclosures}

Financial support: No financial support was received for this submission.

Conflict of interest: The authors have no conflict of interest.

\section{Bibliografia}

1. Sistema di sorveglianza Okkio alla Salute: sintesi dei risultati 2014. www.epicentro.iss.it/okkioallasalute.

2. NIH conference. Gastrointestinal surgery for severe obesity. Consensus Development Conference Panel. Ann Intern Med. 1991;115(12):956-61.

3. Clinical Guidelines on the Identification, Evaluation and Treatment of Overweight and Obesity in Adults. The Evidence Report. National Institutes of Health. Obes Res. 1998;6(Suppl. 2): 51S-209S

4. Adams TD, Gress RE, Smith SC, et al. Long-term mortality after 
gastric bypass surgery. N Engl J Med. 2007;357:753-61.

5. Indagine conoscitiva SICOb (Società Italiana Chirurgia dell'Obesità).

6. Rubino F, Forgione A. The mechanism of diabetes control after gastrointestinal by-pass surgery reveals a role of the proximal small intestine in the pathophysiology of type-2 diabetes. Ann Surg. 2006;244(5):741-9.

7. Kadera BE, Portenier DD, Yurcisin BM, et al. Evidence for a metabolic mechanism in the improvement of type 2 diabetes after sleeve gastrectomy in a rodent model. Surg Obes Relat Dis. 2013;9(3):447-52.

8. Abbatini F, Capoccia D, Casella G, Soricelli E, Leonetti F, Basso $\mathrm{N}$. Long-term remission of type 2 diabetes in morbidly obese patients after sleeve gastrectomy. Surg Obes Relat Dis. 2013;9(4):498-502.

9. Basso N, Capoccia D, Rizzello M, et al. First-phase insulin secretion, insulin sensitivity, ghrelin, GLP-1, and PYY changes $72 \mathrm{~h}$ after sleeve gastrectomy in obese diabetic patients: the gastric hypothesis. Surg Endosc. 2011;25(11):3540-50.

10. Iseki K, Ikemiya Y, Kinjo K, et al. Body mass index and the risk of development of end-stage renal disease in a screened cohort. Kidney Int. 2004;65:1870-6.

11. Verani RR. Obesity-associated focal segmental glomerulosclerosis: pathological features of the lesion and relationship with cardiomegaly and hyperlipidemia. Am J Kidney Dis. 1992;20:629-34.

12. Amann K, Benz K. Structural renal changes in obesity and diabetes. Sem Nephrol. 2013;33:23-33.

13. Semins MJ, Asplin JR, Steele K, et al. The effect of restrictive bariatric surgery on urinary stone risk factors. Urology. 2010;76:826-9.

14. Praga M, Hernandez E, Herrero JC, et al. Influence of obesity on the appearance of proteinuria and renal insufficiency after unilateral nephrectomy. Kidney Int. 2000;58:2111-8.

15. McTigue K, Larson JC, Valoski A, et al. Mortality and cardiac and vascular outcomes in extremely obese women. JAMA. 2006;296:79-86.

16. Rao BB, Bhattacharya A, Agrawal V. Renal outcome of bariatric surgery in obese adults with diabetic kidney disease. J Nephrol. 2014;27:361-70.

17. Kawar B, Bello AK, El Nahas AM. High prevalence of microalbuminuria in the overweight and obese population: data from a UK population screening programme. Nephron Clin Pract. 2009;112:c205-12.

18. Gill H, Whaley-Connell A, Mugo M, et al. The central role of insulin resistance in the metabolic syndrome. Am J Med Sci. 2005;330:290-4.

19. Sowers JR. Insulin resistance and hypertension. Am J Physiol Heart Circ Physiol. 2004;286: H1597-602.

20. Coward RJ, Welsh GI, Yang J, et al. The human glomerular podocyte is a novel target for insulin action. Diabetes. 2005;54:3095-102.

21. Sarafidis PA, Whaley-Connell A, Sowers JR, et al. Cardiometabolic syndrome and chronic kidney disease: what is the link? J Cardiometab Syndr. 2006;1:58-65.

22. Chen S, Jim B, Ziyadeh FN. Diabetic nephropathy and transforming growth beta: transforming our view of glomerulosclerosis and fibrosis build-up. Semin Nephrol. 2003;23: 532-43.

23. Ix JH, Sharma K. Mechanisms linking obesity, chronic kidney disease, and fatty liver disease: the role of fetuin-A, adiponectin, and AMPK. JASN. 2010;21(3):406-12.

24. Hunley TE, Ma LJ, Kon V. Scope and mechanisms of obesity-related renal disease. Curr Opin Nephrol Hypertens. 2010;19:227-34.
25. Kamijo A, Kimura K, Sugaya $T$, et al. Urinary free fatty acids bound to albumin aggravate tubulointerstitial damage. Kidney Int. 2002;62:1628-37.

26. Hajer GR, van HaeftenTW, Visseren FL. Adipose tissue dysfunction in obesity, diabetes, and vascular diseases. Eur Heart J. 2008;29(24):2959-71.

27. Nagase M, Fujita T. Aldosterone and glomerular podocyte injury. Clin Exp Nephrol. 2008;12:233-42.

28. Rüster $\mathrm{C}$, Wolf $\mathrm{G}$. The role of the renin-angiotensin-aldosterone system in obesity-related renal diseases. Semin Nephrol. 2013; 33:44-53.

29. Wolf $G$, Chen S, Han DC, et al. Leptin and renal disease. Am J Kidney Dis. 2002;39:1-11.

30. Wolf G, Hamann A, Han DC, et al. Leptin stimulates proliferation and TGF-beta expression in renal glomerular endothelial cells: potential role in glomerulosclerosis. Kidney Int. 1999;56:860-72.

31. Sharma K, Ramachandrarao S, Qiu G, et al. Adiponectin regulates albuminuria and podocyte function in mice. J Clin Invest. 2008;118:1645-56.

32. Sjostrom L, Peltonen $M$, Jacobson $P$, et al. Bariatric surgery and long-term cardiovascular events. JAMA. 2012;307:56-65.

33. Ahmed $\mathrm{MH}$, Byrne $\mathrm{CD}$. Bariatric surgery and renal function: a precarious balance between benefit and harm. Nephrol Dial Transplant. 2010;25:3142-7.

34. MCampos G, Rabl C, Roll GR, et al. Better weight loss, resolution of diabetes, and quality of life for laparoscopic gastric bypass vs banding. Arch Surg. 2011;146(2):149-55.

35. Lee WJ, Chong K, Ser KH, et al. Gastric bypass vs sleeve gastrectomy fot type 2 dibetes mellitus. Arch Surg. 2011;146(2):143-8.

36. Sjostrom L, Peltonen M, Jacobson $\mathrm{P}$, et al. Association of bariatric surgery wiyh long-term remission of type 2 diabetes and wiyh microvascular and macrovascular complications. JAMA. 2014;311(22):2297-304.

37. Brochner-Mortensen J, Rickers H, Balslev I. Renal function and body composition before after intestinal bypass operation in obese patients. Scand J Clin Lab Invest. 1980;40:695-702.

38. Changac A, Weinstein T, Herman M, Hirsh J, Gafter U, Ori Y. The effects of weight loss on renal function in patients with severe obesity. J Am Soc Nephrol. 2003;14:1480-6.

39. Serra A, Granada ML, Romero R, et al. The effect of bariatric syrgery on adipocycytokines, renal parameters and other cardiovascular risk factors in severe and very severe obesity: Oneyear follow up. Clin Nut. 2006;25:400-8.

40. Navarro-Díaz M, Serra A, Romero R, et al. Effect of drastic weight loss after bariatric surgery on renal parameters in extremely obese patients: long-term follow-up. J Am Soc Nephrol. 2006;17:S213-7.

41. Bolignano D, Zoccali C. Effects of weight loss on renal function in obese CKD patients: a systematic review. Nephrol Dial Transplant. 2013;28(Suppl. 4):iv82-98.

42. Navaneethan SD, Yehnert H. Bariatric surgery and progression of chronic kidney disease. Surg Obes Relat Dis. 2009;5:662-5.

43. Serpa Neto A, Bianco Rossi FM, Dal Moro Amarante R, et al. Effect of weight loss after Roux-en-Y gastric bypass, on renal function and blood pressure in morbidly obese patients. J Nephrol. 2009;22:637-46.

44. Alexander JW, Goodman HR, Hawver LR, et al. Improvement and stabilization of chronic kidney disease after gastric bypass. Surg Obes Relat Dis. 2009;5:237-24.

45. Schuster DP, Teodorescu M, Mikami D, et al. Effect of bariatric surgery on normal and abnormal renal function. Surg Obes Relat Dis. 2011;7:459-4.

46. Carlsson LM, Romeo $\mathrm{S}$, Jacobson $\mathrm{P}$, et al. The incidence of albuminuria after bariatric surgery and usual care in Swedish Obese Subjects (SOS): a prospective controlled intervention trial. Int J 
Obes. 2015;39(1):169-75.

47. Luaces M, Martínez-Martínez E, Medina M, et al. The impact of bariatric surgery on renal and cardiac functions in morbidly obese patients. Nephrol Dial Transplant. 2012;27(Suppl. 4):iv53-7.

48. Sinha MK, Collazo-Clavell ML, Rule A, et al. Hyperoxaluric nephrolithiasis is a complication of Roux-en-Y gastric bypass surgery. Kidney Int. 2007;72:100-7.

49. Lieske JC, Mehta RA, Milliner DS, Rule AD, Bergstralh EJ, Sarr MG. Kidney stones are common after bariatric surgery. Kidney Int. 2015;87(4):839-45.

50. Asplin JR, Coe FL. Hyperoxaluria in kidney stone formers treated with modern bariatric surgery. J Urol. 2007;177:565-9.

51. Nasr SH, D'Agati VD, Said SM, et al. Oxalate nephropathy complicating Roux-en-Y Gastric Bypass: an underrecognized cause of irreversible renal failure. Clin J Am Soc Nephrol. 2008;3:1676-83.

52. Kelly JP, Curhan GC, Cave DR, Anderson TE, Kaufman DW. Factors related to colonization with Oxalobacter formigenes in U.S. adults. J Endourol. 2011;25(4):673-9.

53. Jáuregui-Lobera I. Iron deficiency and bariatric surgery. Nutrients. 2013;(5):1595-608.

54. Malinowski S. Nutritional and metabolic complications of bar- iatric surgery. Am J Med Sci. 2006;331:219-25.

55. Gasteyger C, Suter M, Gaillard RC, Giusti V. Nutritional deficiencies after Roux-en-Y gastric bypass for morbid obesity often cannot be prevented by standard multivitamin supplementation. Am J Clin Nutr. 2008;87:1128-33.

56. Sinha N, Shieh A, Stein EM, et al. Increased PTH and 1.25(OH) (2)D levels associated with increased markers of bone turnover following bariatric surgery. Obesity. 2011;19:2388-93.

57. Abegg K, Gehring N, Wagner CA, et al. Roux-en-Y gastric bypass surgery reduces bone mineral density and induces metabolic acidosis in rats. Am J Physiol Regul Integr Comp Physiol. 2013;305:R999-1009.

58. Costas Fourtounas. Tranplant options for patients with type 2 diabetes and chronic kidney disease. World J Transplant. 2014;4(2):102-10.

59. Hill CJ, Courtney $A E$, Cardwell $C R$, et al. Recipient obesity and outcomes after kidney transplantation: a systematic review and meta-analysis. Nephrol Dial Transplant. 2015;30(8): 1403-11.

60. Ziemiański P, Lisik W, Marszałek RJ, et al. Improvement of graft function following roux-en-Y gastric bypass surgery in a morbidly obese kidney recipient: a case report and literature review. Ann Transplant. 2014;19:639-42. 